\title{
Expressions of miRNA29 target genes CCND2 and CDK6 in cervical cancer
}

\author{
Shuna Shen, Hui Wang; Benli Gong \\ Department of Obstetrics and Gynecology, The Third Affiliated Hospital of Inner Mongolia Medical University, Baotou, Inner \\ Mongolia, China
}

Received: January 13, 2018

DOI: $10.14725 /$ dcc.v5n1p14

Accepted: February 19, $2018 \quad$ Online Published: March 10, 2018

URL: http://dx.doi.org/10.14725/dcc.v5n1p14

\begin{abstract}
Objective: To study the expression of miRNA29 target genes recombinant cyclin D2 (CCND2) and cyclin-dependent kinase 6 (CDK6) in cervical squamous cell carcinoma tissues and their relationship with clinicopathological factors.

Methods: Levels of mRNA of CCND2 and CDK6 in cervical squamous cell carcinoma tissues, cervical intraepithelial neoplasia (CIN) tissues and normal cervical tissues were detected by reverse transcription-polymerase chain reaction (RT-PCR).

Results: There was a statistical difference in the expression of CCND2 (one of miRNA29 target genes) in normal cervical tissues, CIN tissues and cervical squamous cell carcinoma tissues $(\mathrm{H}=29.27, p=.00)$, but there was no statistical difference in the expression of CDK6 (one of miRNA29 target genes) in them $(\mathrm{H}=2.76, p=.25)$. CCND2 was positively correlated to CDK6 in CIN tissues $(r=0.58, p<.05)$.

Conclusions: CCND2, one of miRNA29 target genes, may be involved in the occurrence and development of cervical cancer, but CDK6 is less relevant to the occurrence and development of cervical cancer; CCND2 and CDK6 may play a synergistic role in the occurrence and development of CIN.
\end{abstract}

Key Words: Cervical cancer, Recombinant cyclin D2, Cyclin-dependent kinase 6, MicroRNA29

Cervical cancer is a common malignant tumor in gynecology. The out-of-control in cell cycle has a significant correlation with the occurrence of tumors. ${ }^{[1,2]}$ A miRNA is a set of small, endogenous, non-coding, single-chain RNA molecules, which are widely present in eukaryotic organisms. Whereas, microRNA29 (miRNA29) is lowly expressed in a variety of malignant tumors in a way that targeting recombinant cyclin D2 (CCND2) and cyclin-dependent kinase 6 (CDK6) are involved in the regulation of cell cycle and the occurrence and development of cervical cancer. ${ }^{[3,4]}$ Reverse transcription-polymerase chain reaction (RT-PCR) was applied to this study, in order to detect the expression of CCND2 and CDK6 in cervical squamous cell carcinoma tissues and analyze their interaction in the occurrence and development of cervical cancer.

\section{Materials and methods}

\subsection{Materials}

20 cases of surgical excision specimens due to cervical cancer, 24 cases of cervical conization specimens due to cervical intraepithelial neoplasia (CIN) and 20 cases of panhysterectomy specimens due to hysteromyoma were collected in the Third Affiliated Hospital of Inner Mongolia Medical University from June 2015 to January 2017. All the cases were confirmed by pathological examinations. Patients with

\footnotetext{
*Correspondence: Hui Wang; E-mail: dcc67@ncspress.com; Address: Department of Obstetrics and Gynecology, The Third Affiliated Hospital of Inner Mongolia Medical University, Baotou, Inner Mongolia, China.
} 
cervical cancer (diagnosed as cervical squamous cell carcinoma by pathological results) did not receive radiotherapy and chemotherapy before surgery. According to age, they were divided into two groups: 16 cases of patients were no less than 40 years old; 4 cases of patients were less than 40 years old. According to clinical staging, these patients can be divided as follows: Group I, 6 cases; Group II, 14 cases. From the perspective of lymph node metastasis, they can be divided into two groups: 2 cases with lymph node metastasis and 18 cases without lymph node metastasis. In line with histopathological grading, there are 9 cases with moderate or poor differentiation and 11 cases with high differentiation.

\subsection{Research methods}

In this experiment, fresh specimens were all obtained by pathologists. The wanted tissues were collected immediately after surgery in vitro, with the blood immediately rinsed out by normal saline, placed in cool tissue tubes, immediately frozen in liquid nitrogen, and then stored in the refrigerator until used. RT-PCR was used to detect levels of messenger RNA (mRNA) of CCND2 and CDK6 in cervical squamous cell carcinoma tissues, CIN tissues and normal cervical tissues.

\subsection{Statistical methods}

Sigma State software was used to make a statistical process of data. The comparison in the measurement data among groups was made by Kruskal-Wallis test. The comparison between two groups was made by Mann-Whitney test. Spearman's correlation analysis was applied to the correlation analysis of CCND2 and CDK6. Two-sided test was used, with the test level of $\alpha=0.05$, and the difference was statistically significant $(p<.05)$.

\section{Results}

\subsection{The expression of miRNA29 target gene CCND2 in the three groups}

The average relative expression levels of miRNA29 target gene CCND2 in normal cervical tissues, CIN tissues and cervical squamous cell carcinoma tissues were 0.00140859 , 0.00204650 and 0.01702534 respectively. After the statistical analysis, the expression level of CCND2 in cervical squamous cell carcinoma tissues was significantly higher than that in CIN tissues, with a statistically significant difference $(\mathrm{U}=60.00, p<.05)$. However, there was no statistically significant difference in the expression level of CCND2 in normal cervical tissues and CIN tissues $(\mathrm{U}=184.00, p>.05)$. The expression level of CCND2 in cervical squamous cell carcinoma tissues was significantly higher than that in normal cervical epithelial tissues, and the difference was statistically significant $(\mathrm{U}=16.00, p<.05)$. The results were shown in Table 1 and Figure 1.

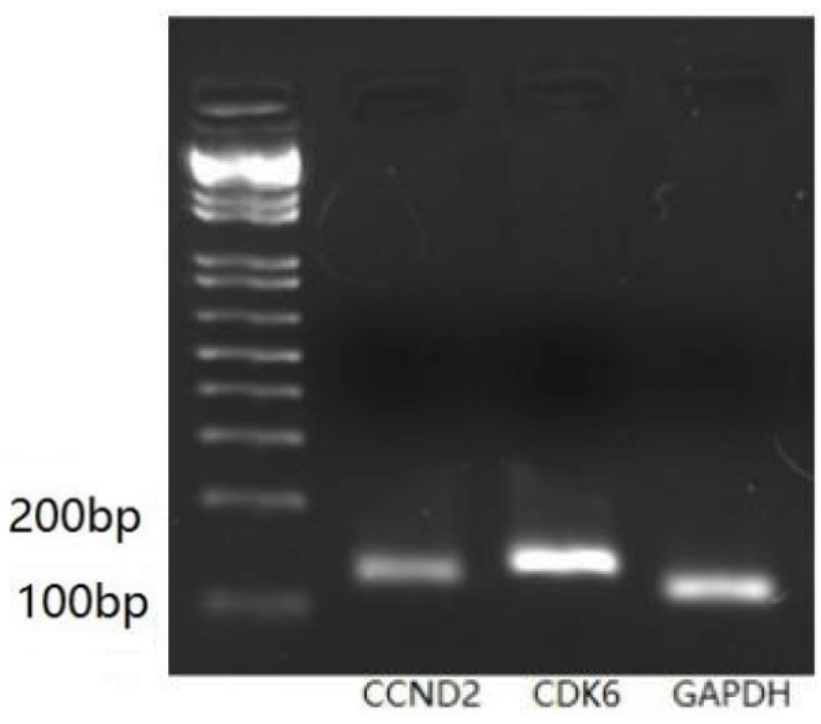

Figure 1: Electrophoretogram of PCR products of CCND 2 and CDK 6 in cervical squamous cell carcinoma tissues

\subsection{The expression of miRNA29 target gene CDK6 in the three groups}

The average relative expression levels of miRNA29 target gene CDK6 in normal cervical tissues, CIN tissues and cervical squamous cell carcinoma tissues were 0.00104340, 0.00028625 and 0.00066675 respectively. However, there was no statistical difference in the expression of CDK6 in them $(\mathrm{H}=2.76, p>.05)$. The results were shown in Table 2 and Figure 1.

Table 1: The expression of CCND2 in normal cervical tissues, CIN tissues and cervical squamous cell carcinoma tissues

\begin{tabular}{|c|c|c|c|c|c|c|c|}
\hline & Group & Cases (n) & CCND2 Mean Value & $\mathbf{H}$ & $p_{1}$ & $\mathbf{U}$ & $p_{2}$ \\
\hline 1 & Cervical Squamous Cell Carcinoma Group & 20 & 0.01702534 & & & 60.00 & $<.05^{*}$ \\
\hline 2 & CIN Group & 24 & 0.00204650 & 29.27 & $<.05$ & 184.00 & $>.05^{* *}$ \\
\hline 3 & Normal Group & 20 & 0.00140859 & & & 16.00 & $<.05^{* * *}$ \\
\hline
\end{tabular}

Note. $p_{1}$ stands for the comparison among three groups, and $p_{2}$ stands for the comparison between two groups; " represents the comparison between Group 1 and Group $2,{ }^{* *}$ represents the comparison between Group 2 and Group 3, and ${ }^{* * *}$ represents the comparison between Group 1 and Group 3 
2.3 Correlation of miRNA29 target genes CCND2 and CDK6 to the occurrence and development of cervical cancer

Spearman's correlation analysis showed that: In CIN tissues, the expression of CCND2 was positively correlated to the expression of CDK6. The results were statistically significant $(r=0.58, p<.05)$. In addition, correlation analysis of CCND2 and CDK6 in cervical squamous cell carcinoma tissues was not statistically significant $(p>.05)$.

2.4 The comparison in clinicopathological characteristics of miRNA-regulated CCND2 in cervical cancer tissues

It was found by a further analysis of the relationship between CCND2 expression and clinicopathological charac- teristics in cervical squamous cell carcinoma tissues that, the difference between CCND2 expression and the degree of pathological differentiation was of statistical significance $(p<.05)$; As to lymph node metastasis, it cannot be compared due to the uneven distribution of lymph nodes. There was no statistical significance in the difference of comparison in FIGO staging and age ( $p>.05$, see Table 3$)$.

\subsection{The expression of miRNA29 target gene CCND2 in CIN tissues}

CIN group can be subdivided into two groups: CIN I/II group and CIN III group. The results showed that the expression of CCND2 in CIN III group was significantly higher than that in CIN I/II group, and the difference was of statistical significance $(p<.05$, see Table 4$)$.

Table 2: The expression of CDK6 in normal cervical tissues, CIN tissues and cervical squamous cell carcinoma tissues

\begin{tabular}{llllll}
\hline & Group & Cases (n) & CDK6 Mean Value & H & $\boldsymbol{p}$ \\
\hline 1 & Cervical Squamous Cell Carcinoma Group & 20 & 0.00066675 & & \\
2 & CIN Group & 24 & 0.00028625 & 2.76 & $>.05$ \\
3 & Normal Group & 20 & 0.00104340 & & \\
\hline
\end{tabular}

Table 3: The relationship between the expression of CCND2 and clinicopathological characteristics in cervical squamous cell carcinoma tissues

\begin{tabular}{|c|c|c|c|c|}
\hline & Cases (n) & CCND2 Mean Value & $\mathbf{U}$ & $\bar{p}$ \\
\hline \multicolumn{5}{|l|}{ Age (years old) } \\
\hline - $\geq 40$ & 14 & 0.01957248 & \multirow{2}{*}{39.00} & \multirow{2}{*}{$>.05$} \\
\hline - $<40$ & 6 & 0.01108203 & & \\
\hline \multicolumn{5}{|l|}{ Lymph Node Metastasis } \\
\hline - Positive & 2 & 0.02367749 & \multirow{2}{*}{-} & \multirow{2}{*}{-} \\
\hline - Negative & 18 & 0.01628622 & & \\
\hline \multicolumn{5}{|l|}{ FIGO Staging } \\
\hline - $\mathrm{I}$ & 6 & 0.01002174 & \multirow{2}{*}{23.00} & \multirow{2}{*}{$>.05$} \\
\hline - II & 14 & 0.02002689 & & \\
\hline \multicolumn{5}{|c|}{ Degree of Histopathological Differentiation } \\
\hline - High Differentiation & 11 & 0.00741729 & \multirow{2}{*}{23.00} & \multirow{2}{*}{$<.05$} \\
\hline - Moderate or Poor Differentiation & 9 & 0.028768521 & & \\
\hline
\end{tabular}

Table 4: The expression of CCND2 in CIN tissues

\begin{tabular}{lllll}
\hline & Cases (n) & CCND2 Mean Value & U & $p$ \\
\hline CIN I/II & 13 & 0.00117632 & 4.00 & $<.05$ \\
CIN III & 11 & 0.00327582 & & \\
\hline
\end{tabular}




\section{Discussion}

\section{1 miRNA29 and malignant neoplasms}

miRNA29 is one of the members of miRNA and it is abnormally expressed in various cancers, such as breast cancer, gastric carcinoma and cervical cancer, etc. miRNA29 is involved in the regulation of cell cycle by targeting CCND2, so as to inhibit the occurrence of cervical cancer. In cervical cancer tissues, CDK6, one of target genes for miRNA29, participates in the occurrence of cervical cancer by regulating cell cycle.

\subsection{CCND2 and cervical neoplasms}

CCND2, a member of D-type cyclins, plays a key role in cell cycle regulation, differentiation and malignant transformation. ${ }^{[4]}$ Zou DL et al. ${ }^{[5]}$ found that the expression of CCND2 was higher in cervical cancer tissues, and miRNA29 was involved in the occurrence and development of cervical cancer by targeting CCND2. The results of this study indicated that the expression of CCND2 in cervical squamous cell carcinoma tissues was higher than that in normal cervical tissues and CIN tissues, with the difference statistically significant, and it was related with the pathological grading; The difference in the expression of CCND2 in normal cervical tissues and CIN tissues was of no statistical significance. However, there was a difference in the expression of CCND2 in CIN I/II and CIN III groups. The difference was statistically significant. The results of this experiment indicated that, CCND2, one of miRNA29 target genes, was not significantly abnormally expressed in the early cervical lesions (CIN I/II group). It may promote the growth of tumor cells with the progression of cervical lesions coming into play. Since pathological grading is an important indicator for prognosis of malignant tumors, CCND2 has a critically clinical significance in the prognosis evaluation of cervical cancer.

\subsection{CDK6 and cervical neoplasms}

CDK6, a member of CDK family, is induced by mitogens to bind cyclin D and participate in the regulation of cell cycle. Currently, it is found that the activity of CDK6 is enhanced in various tumors due to CDK6 gene amplification, over-expression or deficiency in cell cycle inhibitors. ${ }^{[6]}$

\section{References}

[1] Li Y, Liu J, Liu ZZ, et al. MicroRNA-145 inhibits tumor growth and metastasis in osteosarcoma by targeting cyclin-dependent kinase, CDK6. Eur Rev Med Pharmacol Sci. 2016; 20(24): 51175125. PMid: 28051259.

[2] Zhu K, Liu L, Zhang J, et al. MiR-29b suppresses the prolifera-
miRNA29 may be the one that is the most related with HPV carcinogenesis in the miRNA family. It may participate in the HPV-associated process of cervical cancer through some biological functions such as cell cycle, suggesting that miRNA29 may be a miRNA that is related to the suppression of cervical squamous cell carcinoma. Wang $\mathrm{H}$ et

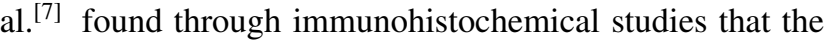
expression of CDK6 protein in cervical cancer tissues was significantly higher. The experimental results showed that the difference in the expression of CDK6 in normal cervical tissues, CIN tissues, and cervical squamous cell carcinoma tissues was of no statistical significance, which was inconsistent with literature reports. This result may be due to the fact that CDK6 is only part of the signal transduction system with a large cell cycle, and this study is deficient in the amount of specimens. Therefore, it is necessary to further expand the amount of specimens and quantitatively detect the level of protein, so as to determine the effect of miRNA29 target gene CDK6 in the occurrence and development of cervical cancer.

\subsection{Correlation of CCND2 and CDK6 to cervical neoplasms}

In this study, RT-PCR was used to detect the expression of CCND2 and CDK6 in CIN tissues of cervical cancer and statistical methods were applied to the correlation analysis of these two factors. The results showed that the expression levels of CCND2 and CDK6 were not correlated in cervical squamous cell carcinoma tissues but positively correlated in CIN tissues, suggesting that miRNA29 target genes CCND2 and CDK6 may play a synergistic role in CIN tissues. The mechanism of miRNA29 in the occurrence and development of cervical cancer was further explored by this study under the condition of large sample size. With the occurrence and development of cervical cancer, studies on the relationship of miRNA29 target genes CCND2 and CDK6 need to be continued to further explore the mechanism of miRNA29 in the occurrence and development of cervical cancer, and provide a theoretical basis for miRNA29 in the diagnosis and treatment of cervical cancer.

\section{Conflicts of Interest Disclosure}

The authors have no conflicts of interest related to this article.

tion and migration of osteosarcoma cells by targeting CDK6. Protein Cell. 2016; 7(6): 434-444. PMid: 27230400. https://doi. org/10.1007/s13238-016-0277-2

[3] Zhu HQ. Cyclin-dependent kinase 6 and tumor \& cell differentiation. Chemistry of Life. 2009; 28(1): 52-55.

[4] Fan Z, Cui H, Xu X, et al. MiR-125a suppresses tumor growth, invasion and metastasis in cervical cancer by targeting STAT3. On- 
cotarget. 2015; 6(28): 25266-25280. PMid: 26389681. https: //doi.org/10.18632/oncotarget. 4457

[5] Zou DL, Wang D, Zhou Q. The mechanism of microRNA-29b regulating cyclin D2 expression in the genesis of human cervical cancer. Tumor. 2014; 34(12): 1090-1096.

[6] Handschick K, Beuerlein K, Jurida L, et al. Cyclindependent ki- nase 6 is a chromatin-bound cofactor for NF- $\kappa \mathrm{B}$-dependent gene expression. Mol Cell. 2014; 53(2): 193-208. PMid: 24389100. https://doi.org/10.1016/j.molcel.2013.12.002

[7] Wang H, Shen SN, Wang JF. A study on expression of CDK6 in cervical malignant tumor tissue. China Journal of Modern Medicine. 2013; 23(34): 60-62. 